\title{
Intramural Hematoma as Unexpected Complication of COVID-19 Infection
}

\author{
Francesca Terzi, MD ${ }^{1}$ Mariano Cefarelli, MD ${ }^{2}$ Rossella Fattori, MD, PhD ${ }^{3}$ Marco Di Eusanio, MD, PhD ${ }^{2}$ \\ ${ }^{1}$ Cardiology Unit, Azienda Ospedaliera Ospedali Riuniti Marche Nord, \\ Pesaro, Italy \\ ${ }^{2}$ Cardiac Surgery Unit, Lancisi Cardiovascular Center, Polytechnic \\ University of Marche, Ancona, Italy \\ ${ }^{3}$ Cardiovascular Center, Marfan Center, Ospedali Riuniti Marche, \\ Ancona, Italy \\ Address for correspondence Francesca Terzi, MD, Cardiology Unit, \\ Azienda Ospedaliera Ospedali Riuniti Marche Nord Pesaro, PU, \\ Piazzale Cinelli 4, 61121 Italy (e-mail: francesca.terzi3@gmail.com). \\ AORTA 2020;8:74-75.
Abstract
Keywords
- intramural hematoma
- COVID-19
- aorta
- inflammation
- cytokine storm \\ Novel coronavirus disease-2019 (COVID-19) is an ominous infectious disease that \\ seems capable to attack any organ system, leading in the most severe cases to patient \\ death. COVID-19 has been associated with multiple cardiovascular complications of \\ inflammatory and immune origin, leading to a wide spectrum of vascular damage, \\ myocardial injury, stroke, and pulmonary obstruction. We report the case of a patient \\ with COVID-19 infection who developed an acute aortic syndrome with the character- \\ istics of aortic intramural hematoma.
}

\section{Introduction}

Patients with preexisting cardiovascular disease (CVD) have an increased risk of severe complications from novel coronavirus disease 2019 (COVID-19). However, even COVID-19 patients without a history of CVD may be at risk for cardiovascular complications, ${ }^{1,2}$ often constituting the primary cause of death.

Aortic intramural hematoma (IMH) is an important subgroup of acute aortic syndrome (AAS) and may account for 10 to $25 \%$ of them. When it involves the ascending aorta, it is highly lethal and requires emergent surgery. ${ }^{3}$ Nuclear imaging and biomarker-based studies have suggested a strong association between aortic wall inflammation and IMH, providing information on its risk stratification. ${ }^{4,5}$

Here, we present the case of a patient with COVID-19 infection complicated with IMH who underwent emergency surgery.

\section{Case Presentation}

A 73-year-old Italian man, with an unremarkable clinical history and no cardiovascular risk factors, was admitted to the emergency department with chest pain and shortness of

received

April 23, 2020

accepted after revision

May 11,2020 breath, along with lower limb paresthesia. The patient disclosed a 10-day history of fever, cough, and sore throat, treated with paracetamol $2 \mathrm{~g} /$ day. Based on real-time polymerase chain reaction analysis of a sputum sample, he was diagnosed with COVID-19.

On presentation, he was normotensive; heart rate was $60 \mathrm{bpm}$ and body temperature was $38^{\circ} \mathrm{C}$. Laboratory tests demonstrated high inflammatory markers and lymphopenia (white blood cell count, $20.070 / \mathrm{mm}^{3}$; neutrophils, $92 \%$; lymphocytes, $1.5 \%$; and C-reactive protein, $20.50 \mathrm{mg} / \mathrm{dL}$ ), increased high sensitive Troponin I ( $200 \mathrm{ng} / \mathrm{L}$ ), and N-terminal pro-brain natriuretic peptide $(4,000 \mathrm{pg} / \mathrm{mL})$. Even interleukin (IL)-6 level was elevated $(121 \mathrm{pg} / \mathrm{mL})$. Estimated glomerular filtration rate and liver enzymes were unremarkable. Chest contrast-enhanced computed tomography (CT) revealed multiple ground-glass opacities with bilateral parenchymal consolidation and interlobular septal thickening suggestive for COVID-19 interstitial pneumonia (-Fig. 1). Moreover, CT showed an IMH extending from the ascending aorta (supravalvular plane) to the abdominal aorta. The aorta was normal in dimension (Valsalva's sinuses, $37 \mathrm{~mm}$; ascending aorta, $36 \mathrm{~mm}$; aortic arch, $30 \mathrm{~mm}$ ). Tel: +1(212) 760-0888.
Copyright $(2020$ by Thieme Medical Publishers, Inc., 333 Seventh Avenue, New York, NY 10001, USA.

License terms

$10.1055 / \mathrm{s}-0040-1713107$ ISSN 2325-4637.

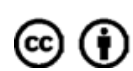




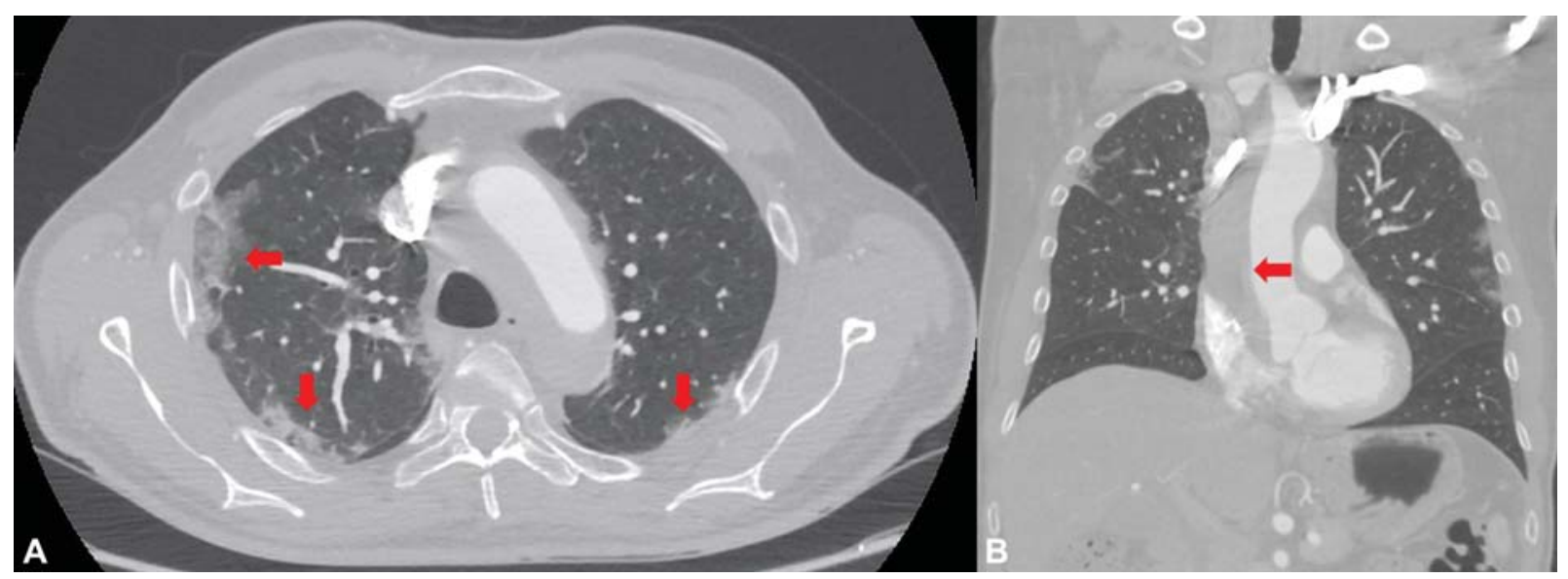

Fig. 1 Computed tomography scan of the chest: (A) multiple ground-glass opacities with bilateral parenchymal consolidation and interlobular septal thickening (red arrows) (B) intramural hematoma of ascending aorta (red arrow) with periadventitial aortic wall enhancement.

The patient was transferred urgently to a referral hospital for cardiac surgery. The aorta and the heart were exposed through a median sternotomy. Under moderate hypothermic circulatory arrest $\left(28^{\circ} \mathrm{C}\right.$ nasopharyngeal temperature) and antegrade selective cerebral perfusion (ASCP), the ascending aorta and hemiarch were replaced by means of a $28 \mathrm{~mm}$ straight vascular graft. cardiopulmonary bypass, crossclamp, and ASCP times were, 58, 40, and 10 minutes, respectively. All team members were protected with an N95 respirator mask and wore double gowns, gloves, and eye protection.

The patient, extubated on postoperative day (POD) 1 , experienced respiratory insufficiency requiring reintubation 3 days later and remained under ventilation for 7 days. Noninvasive ventilation therapy was applied during the 72 hours following reextubation with beneficial effects. For the concomitant COVID-19 disease, the patient received empiric treatment with hydroxychloroquine and lopinavir/ ritonavir. Before transferring the patient to the referral hospital on POD 19, the patient transited from the intensive care unit (ICU) through our COVID-19 mid- and low-intensive care units. At discharge, the COVID-19 test was still positive. The patient is now following a rehabilitation program and two consecutive COVID-19 tests are negative.

\section{Discussion}

Cardiovascular involvement has been reported in patients with COVID-19; inflammation in the vascular system can result in diffuse microangiopathy, thrombosis, and vascular damage. ${ }^{1}$ Inflammation of the aortic wall also seems to play a role in the pathogenesis of AAS and in particular of IMH. ${ }^{7}$ Several previous studies have suggested that inflammation exists in the involved aorta in patients with classic $\mathrm{AD}^{5-8}$ and IMH. $^{7}$ Our COVID-19 patient without cardiovascular risk factors complicated by IMH could intriguingly indicate IMH as another potential COVID-19 major vascular complication; the devastating systemic inflammatory response to COVID-19 infection with the typical "cytokine storm" could likely represent the underlying mechanism of the aortic wall damage. However, only the evolving understanding of the multiple mechanisms in which the virus, or the immune response to it, attacks human cells will lead to a better comprehension and treatment of the protean manifestations of COVID-19 disease.

\section{Funding \\ None}

\section{Conflict of Interest}

The authors declare no conflict of interest related to this article.

\section{Acknowledgment}

None.

\section{References}

1 Driggin E, Madhavan MV, Bikdeli B, et al. Cardiovascular considerations for patients, health care workers, and health systems during the coronavirus disease 2019 (COVID-19) pandemic. J Am Coll Cardiol 2020; 75(18):. Doi: 10.1016/j.jacc.2020.03.031

2 Zheng YY, Ma YT, Zhang JY, Xie X. COVID-19 and the cardiovascular system. Nat Rev Cardiol 2020;17(05):259-260

3 Evangelista A, Mukherjee D, Mehta $\mathrm{RH}$, et al; International Registry of Aortic Dissection (IRAD) Investigators. Acute intramural hematoma of the aorta: a mystery in evolution. Circulation 2005;111(08):1063-1070

4 Akamatsu et al. Evaluation of aortic wall inflammation in acute aortic intramural hematoma using FDG-PET/CT in relation to prognostic aorta-related outcome. J Nucl Med 2017;58(01):1167

5 Kuehl H, Eggebrecht H, Boes T, et al. Detection of inflammation in patients with acute aortic syndrome: comparison of FDG-PET/CT imaging and serological markers of inflammation. Heart 2008;94 (11):1472-1477

6 Erbel R, Aboyans V, Boileau C, et al. 2014 ESC Guidelines on the diagnosis and treatment of aortic diseases: document covering acute and chronic aortic diseases of the thoracic and abdominal aorta of the adult. The task force for the diagnosis and treatment of aortic diseases of the European Society of Cardiology (ESC). Eur Heart J 2014;35(41):2873-2926

7 Kitai T, Kaji S, Kim K, et al. Prognostic value of sustained elevated C-reactive protein levels in patients with acute aortic intramural hematoma. J Thorac Cardiovasc Surg 2014;147(01):326-331

8 He R, Guo DC, Estrera AL, et al. Characterization of the inflammatory and apoptotic cells in the aortas of patients with ascending 